Revue d'histoire de l'Amérique française

MA REVUE D.HISTOIRE DE L'AMÉRIQUE FRANÇAISE

\title{
Les sources archivistiques : leur utilisation dans l'étude de la bourgeoisie marchande bas-canadienne (1800-1830)
}

\section{George Bervin}

Volume 38, numéro 2, automne 1984

Bourgeoisies et Petites Bourgeoisies

URI : https://id.erudit.org/iderudit/304260ar

DOI : https://doi.org/10.7202/304260ar

Aller au sommaire du numéro

Éditeur(s)

Institut d'histoire de l'Amérique française

ISSN

0035-2357 (imprimé)

1492-1383 (numérique)

Découvrir la revue

Citer cet article

Bervin, G. (1984). Les sources archivistiques : leur utilisation dans l'étude de la bourgeoisie marchande bas-canadienne (1800-1830). Revue d'histoire de

l'Amérique française, 38(2), 203-222. https://doi.org/10.7202/304260ar d'utilisation que vous pouvez consulter en ligne. 


\title{
LES SOURCES ARCHIVISTIQUES: LEUR UTILISATION DANS L'ÉTUDE DE LA BOURGEOISIE MARCHANDE BAS-CANADIENNE $(1800-1830)^{*}$
}

\author{
GEORGE BERVIN \\ Département d' histoire \\ Université de Montréal
}

\section{Introduction}

Il s'agit d'examiner ici la valeur et l'utilité de certaines sources ${ }^{1}$ dans l'élaboration d'une histoire socio-économique portant sur la bourgeoisie marchande bas-canadienne de la première moitié du XIX ${ }^{\mathrm{e}}$ siècle. Nous mettrons en relief certains actes notariés souvent délaissés ou ignorés par trop de chercheurs, de façon à montrer quel genre de données ils contiennent, de quelle manière on peut les utiliser et quelles sont leurs limites. De plus, nous verrons dans quelle mesure ces différentes sources combinées entre elles peuvent permettre l'élaboration d'hypothèses rigoureuses sur les activités commerciales des grands marchands bas-canadiens de cette période.

Le présent texte revêt donc un caractère méthodologique. Il traite de la rigueur requise pour le maniement des informations livrées par les documents d'archives et de l'utilisation de ces informations dans l'analyse historique. Nous toucherons brièvement aux inventaires après décès, aux actes de procuration, aux livres de comptes, aux actes de vente de biens immobiliers et fonciers, aux registres de vente de terres par le shérif, aux actes d'affrètement de navires, aux obligations et aux quittances, et enfin, aux actes de transport.

* Cet article constitue la version remaniée d'une communication présentée lors du Congrès de l'Institut d'histoire de l'Amérique française, tenu à Compton les 21 et 22 octobre 1983. Nous tenons à remercier Jean-Pierre Wallot, de l'Université de Montréal, Marc Vallières, de l'Université Laval, et France Galarneau, du Dictionnaire biographique du Canada, pour leurs commentaires et suggestions.

Le choix de ces sources repose, en partie, sur l'utilisation que nous en avons faite dans le cadre du groupe de recherche sur l'histoire économique et la culture matérielle du Bas-Canada dans la première moitié du XIX ${ }^{\mathrm{e}}$ siècle, dirigé par Jean-Pierre Wallot, et au cours de recherches personnelles. Voir à ce sujet George Bervin, "Aperçu sur le commerce et le crédit à Québec, 1820-1830», RHAF, 36, 4 (mars 1983): 527-551; «Environnement matériel et activités économiques des conseillers exécutifs et législatifs à Québec, 1810-1830», Bulletin d'histoire de la culture matérielle, 17 (Musée national de l'Homme, Ottawa): printemps 1983: 45-62. 


\section{I- L'inventaire après décès}

\section{a) Les pages de présentation}

L'un des principes de base qui sous-tend de nombreuses recherches historiques est que l'utilisation d'un seul type de documents ne permet pas généralement d'arriver à une analyse précise d'une conjoncture historique. Dans une étude donnée il est peu fréquent, en effet, qu'une source unique suffise à étayer l'hypothèse de base. Cependant, il faut mentionner que l'un des rares documents pouvant, même pris isolément, permettre d'étudier la fortune mobilière d'un individu au moment de sa mort, est l'inventaire après décès ${ }^{2}$. Il donne également une bonne idée de la situation financière de cette personne à son décès.

Certains chercheurs auraient intérêt à se pencher sur les toutes premières pages de l'inventaire après décès, les pages explicatives. Les informations sociologiques sur les composantes de la famille qu'elles contiennent fournissent de multiples renseignements et, jointes aux contrats de mariage ${ }^{3}$, peuvent donner des résultats très probants lors d'une enquête sur la cellule familiale dans le Bas-Canada au cours de la première moitié du $\mathrm{XIX}^{\mathrm{e}}$ siècle.

Nous y trouvons la (ou les) profession(s) du défunt ${ }^{4}$, son origine ethnique, son lieu de résidence et le régime matrimonial qu'il avait choisi. Dans le cas d'un individu qui a été marié (ce qui semble être le cas dans la presque totalité des inventaires après décès retracés), les pages de présentation de l'inventaire indiquent le nom de son épouse (dans l'ensemble, c'est le nom de jeune fille qui est donné) et les prénoms de ses enfants. Par ailleurs, l'acte nous dit s'il s'agit d'un premier, d'un deuxième ou d'un troisième mariage, à la fois pour le défunt et

2 Pour une analyse plus approfondie de l'inventaire après décès se rapporter, entre autres, à l'étude de Gilles Paquet et Jean-Pierre Wallot, «Les inventaires après décès à Montréal au tournant du XIX ${ }^{\mathrm{e}}$ siècle: préliminaires à une analyse», RHAF, 30, 2 (sept. 1976): 163-221. Dans cet article, qui constitue un exemple d'histoire sérielle et quantitative, les auteurs font la démonstration de l'utilité des inventaires après décès dans l'analyse des niveaux de fortune de différents groupes sociaux à Montréal. Jusqu'ici, il s'agit de l'unique étude portant sur le Bas-Canada pour laquelle les inventaires après décès ont été exploités de façon aussi systématique. Une communication donnée par les mêmes auteurs lors du Colloque international d'histoire comparée des sociétés rurales de la France de l'Ouest et du Québec repose elle aussi sur l'utilisation des inventaires après décès. («Structures sociales et niveaux de richesse dans les campagnes du Québec: 1792 1812», Rochefort, 5-8 juil. 1982). Enfin, consulter également l'article de George Bervin, «Espace physique et culture matérielle du marchand-négociant à Québec (1820-1830)», Bulletin d' histoire de la culture matérielle, 14 (Musée national de l'Homme, Ottawa): printemps 1982: 1-19.

3 Sur l'utilisation que l'on peut faire des contrats de mariage en histoire sociale, consulter Adeline Daumard, «Structures sociales et classement socio-professionnel. L'apport des archives notariales au XVIII ${ }^{\mathrm{e}}$ et au XIX ${ }^{\mathrm{e}}$ siècles», Revue historique, 227, 1 (janv.-mars 1982): 139-154; sur les contrats de mariage, voir en particulier les pages 141-144. Il serait utile de lire aussi l'article de Louis Lavallée, "Les archives notariales et l'histoire de la Nouvelle-France», RHAF, 28, 3 (déc. 1974): 385-398.

${ }_{4}$ Il est à noter qu'il existe aussi des inventaires après décès faits pour des femmes. Cependant, vu que la plupart des inventaires après décès de marchands-négociants que nous avons trouvés concernent des hommes, nous avons opté pour le masculin dans notre texte. 
pour son épouse. Il permet, de plus, de connaître la situation matérielle provisoire (c'est-à-dire avant le règlement final de la succession) de la femme, à partir de la «chambre garnie» à laquelle elle a automatiquement droit dès le décès de son conjoint. La «chambre garnie» est allouée d'après les clauses du contrat de mariage et, dès le début de l'inventaire, l'huissier énumère les différentes pièces de mobilier qui reviennent de droit à la veuve, sans pour autant en mentionner la valeur, et les biens sont classés sous la rubrique "pour mémoire».

Les pages de présentation de l'inventaire nous apprennent également si la veuve est l'exécutrice testamentaire et la tutrice des enfants mineurs, et quelle est sa part dans la succession. Évidemment, nous sommes informés sur son origine ethnique, donnée importante dans une étude cherchant à déterminer s'il y a de nombreux mariages inter-ethniques à l'époque entre les membres d'un même groupe social. Cette nouvelle veuve sait-elle lire et écrire ? Si la réponse est négative, le notaire le mentionne à la fin des pages de présentation de l'inventaire, autrement la veuve appose sa signature au bas de la page.

Toujours en ce qui concerne le portrait sociologique de la famille, nous pouvons calculer la moyenne du nombre d'enfants par catégorie socio-professionnelle. Il est aussi possible de savoir si l'épouse avait déjà des enfants avant ce mariage, et l'inventaire indique le nombre total des enfants issus du couple, en plus d'identifier parmi eux ceux qui sont majeurs et ceux qui ne le sont pas. Nous sommes, de plus, à même de déterminer si des filles nées de ce couple sont déjà mariées, car si tel est le cas leur époux signe l'inventaire à leur place. Une telle information peut être utilisée pour reconstituer des liens familiaux, sociaux et ethniques, puisque la profession du gendre est indiquée et que, par le biais de son nom, nous pouvons établir son origine ethnique. Quant aux enfants majeurs mâles, nous pouvons connaître leur profession au moment de la prisée de l'inventaire et voir si ceux qui sont présents peuvent apposer leur signature au bas du document ${ }^{5}$.

Par ailleurs, l'huissier mentionne la part des biens qui revient à chacun des héritiers légaux ou à leurs descendants directs. Dans une étude sur la consolidation ou la dilapidation des fortunes bourgeoises, ces chiffres pourront être utilisés pour évaluer dans quelle mesure, au cours du XIX $\mathrm{X}^{\mathrm{e}}$ siècle, et à l'intérieur d'une région précise, les familles marchandes continuent, par exemple, d'accroître leur fortune, et pour vérifier si celle-ci s'effrite ou non après la mort du père. Dans le cas où

\footnotetext{
5 Tous les enfants majeurs issus de la bourgeoisie marchande que nous avons rencontrés savent lire et écrire. Mais il serait intéressant de vérifier le nombre d'héritiers mâles qui exercent la profession du père, surtout dans le cas des marchands. Cette information pourrait montrer si dans le Bas-Canada la tendance est à la formation de familles marchandes, de père en fils.
} 
un acte de partage ou de donation pour la famille concernée existerait, le travail serait plus facile à faire ${ }^{6}$.

En outre, en relevant systématiquement les noms des témoins, nous serons en mesure d'examiner les relations sociales de la famille. En effet, lors de la préparation de l'inventaire, en particulier en milieu aisé, il y a souvent un ou des témoins, un curateur, un subrogé tuteur, un exécuteur testamentaire, etc. Tous, sans doute, entretiennent des liens très étroits avec la famille du disparu. Parfois, quelques-uns d'entre eux étaient des associés de celui-ci, ou encore, un gendre, un ami intime ou un parent par alliance. En comparant ces noms avec ceux des personnes présentes lors de la signature du contrat de mariage du défunt, nous pourrons esquisser les grandes lignes des relations sociales de sa famille.

\section{b) La première partie de l'inventaire après décès}

La première partie de l'inventaire après décès permet de reconstituer avec exactitude l'intérieur domestique de la personne décédée, que celle-ci ait été un membre de l'élite ou un représentant des couches populaires et rurales ${ }^{7}$. Dans le cas des personnes bien nanties l'huissier fait une description aussi fidèle que possible de la division physique de la maison. Cette description des lieux permet, entre autres choses, de connaître l'architecture de la résidence principale.

Nous avons constaté que, généralement, l'aisance matérielle du défunt se voit par la précision du document. Les inventaires après décès des couches populaires ne sont pas aussi précis que ceux des couches supérieures. Nous avons remarqué qu'assez souvent le notaire ou l'huissier, lorsqu'ill s'agit des couches populaires, répertorie les biens sans nommer la partie de la maison où ils se trouvent. Mais dans tous les cas, à cause de son contenu très varié, la première partie de l'inventaire après décès peut permettre aux historiens d'écrire l'histoire du quotidien ${ }^{8}$ d'une collectivité et, à l'intérieur de cette collectivité, de chaque groupe social. Les chercheurs qui s'intéressent aux artisans, aux travailleurs et aux habitants peuvent y trouver des éléments précieux

\footnotetext{
$6 \quad$ Voir l'article de Louis Michel sur un marchand en Nouvelle-France, dans lequel il utilise comme sources de base l'inventaire après décès et le compte de partage de la succession du défunt. (Louis Michel, «Un marchand rural en Nouvelle-France. François-Augustin Bailly de Messein, 1709-1771», RHAF, 33, 2 (sept. 1979): 215-262.

Les études menées par Gilles Paquet et Jean-Pierre Wallot, que nous avons citées plus haut, prouvent de façon assez nette l'existence d'inventaires après décès dans presque tous les milieux. Voir aussi Yvan Morin, «La représentativité de l'inventaire après décès. L'étude d'un cas: Québec au début du XIX ${ }^{\mathrm{e}}$ siècle», RHAF, 34, 4 (mars 1981): 515-533.

Sur l'histoire du quotidien, se référer à l'oeuvre monumentale de Fernand Braudel, Civilisation matérielle, économie et capitalisme, $X V^{e}-X V I I I^{e}$ siècle (3 tomes, Armand Colin, Paris, 1979), et en particulier, au tome I, Les structures du quotidien: le possible et l'impossible.
} 
pour l'élaboration d'une histoire de la culture populaire ${ }^{9}$. Par exemple, toute étude sur l'habillement peut être facilitée et ceci, même si l'inventaire n'énumère que les biens du défunt. Un chercheur peut analyser l'évolution des tenues vestimentaires (dans le cas d'une histoire sérielle) à partir de périodes préalablement déterminées, faire une étude sur le textile et se demander par exemple si les tissus utilisés sont différents d'un groupe social à l'autre ${ }^{10}$.

L'étude des inventaires après décès permet également de vérifier si l'accumulation de biens mobiliers dans les résidences survient lors d'une conjoncture économique favorable. De plus, en poussant davantage l'analyse, on peut savoir à quel groupe ethnique appartiennent les marchands qui ont tendance à posséder le plus de biens mobiliers ; autrement dit, identifier ceux qui profitent le plus de la conjoncture économique. Il est aussi possible à partir des inventaires de déterminer si le mobilier se différencie d'un groupe ethnique à l'autre. C'est toute la question du style de vie qui se dessine par le biais des objets domestiques. Grâce aux informations sur le mobilier, on peut déceler l'influence d'une culture sur une autre et chercher à savoir si, par exemple, le mobilier est généralement d'inspiration anglaise, si le bois utilisé dans sa fabrication est d'origine locale ou étrangère et déterminer l'apport des artisans locaux.

L'abondance des données disponibles dans l'inventaire après décès rend possible une évaluation rapide de la richesse mobilière globale d'un ménage. Les difficultés surgissent quand on tente de délimiter la contribution de chacun à la formation de ce patrimoine. Nous avons constaté que l'une des lacunes de l'inventaire après décès est qu'il ne permet pas d'isoler distinctement les biens appartenant à l'épouse de ceux de l'époux. Cette situation peut s'expliquer en partie par le régime matrimonial, car lorsque le contrat de mariage est conclu sous le régime de la communauté de biens le notaire ne fait pas de différenciation quant à leur appartenance, sauf pour les «biens propres». Ce problème méthodologique n'est pas facile à résoudre, à cause de plusieurs variables

\footnotetext{
$9 \mathrm{Au}$ sujet de l'utilisation des inventaires après décès dans l'étude des couches populaires urbaines, lire Jacques Bernier, Quelques boutiques de menuisiers et charpentiers au tournant du $X I X^{e}$ siècle, Collection Mercure, 17 (Musées nationaux du Canada, Ottawa, 1976), 72 p., et du même, Les intérieurs domestiques des menuisiers et charpentiers de la région de Québec, 1810 1819, Collection Mercure, 23 (Musées nationaux du Canada, Ottawa, 1977), 83 p. Voir aussi l'article de Jean-Pierre Hardy, «Niveaux de richesse et intérieurs domestiques dans le quartier Saint-Roch à Québec, 1820-1850», Bulletin d' histoire de la culture matérielle, 17 (Musée national de l'Homme, Ottawa): printemps 1983: 63-94, portant sur plusieurs catégories socio-professionnelles issues des couches populaires dans la ville de Québec. David Thiery Ruddel, pour sa part, a fait une analyse du secteur du textile à partir d'inventaires après décès et d'autres documents. L'auteur met en évidence la part prédominante qu'occupent sur les marchés locaux les vêtements en provenance de Grande-Bretagne. (David Thiery Ruddel, «The domestic textile industry in the region and city of Quebec, 1792-1835», Bulletin d' histoire de la culture matérielle, 17 (Musée national de l'Homme, Ottawa): printemps 1983: 95-125).

10 Concernant le textile et l'habillement, consulter David Thiery Ruddel, «The domestic textile industry in the region and city of Quebec, 1792-1835», op. cit.
} 
dont l'une concerne la partie du mobilier que l'épouse amène dans la résidence lors de la formation du couple. Les problèmes posés - ces biens mobiliers proviennent-ils de la succession de ses parents, ou encore, l'épouse a-t-elle les moyens financiers de les acheter? - nécessitent donc la consultation d'autres sources. Or, ces informations sont déterminantes pour évaluer la structure de la fortune mobilière de la nouvelle famille.

Enfin, il faut retenir que toutes les interprétations qu'amènent les pages de présentation et la première partie de l'inventaire après décès ne seront valables que dans la mesure où l'échantillon constitué sera assez représentatif du groupe visé et assez diversifié pour assurer une bonne valeur statistique et qualitative à l'étude. Au niveau de la méthode, seul un échantillon qui tient compte des diverses périodes et de la hiérarchie sociale permettra de dégager les tendances générales d'une catégorie sociale particulière.

\section{II- La valeur de certaines sources dans l'identification des activités économiques de la bourgeoisie marchande}

Nous avons déjà démontré ailleurs ${ }^{11}$ qu'on peut arriver à bien cerner la fortune mobilière d'un marchand-négociant ou d'une catégorie sociale précise. Les données quantifiables tirées de l'inventaire et du procès-verbal de vente peuvent permettre à l'historien de brosser un tableau très évocateur de l'intérieur domestique et du mode de vie du défunt.

Que peut faire un chercheur lorsque vient le moment de se pencher sur l'origine, la composition, la répartition et la mobilité de la richesse d'un marchand pour une période délimitée? Il ne saurait être question pour arriver à des conclusions définitives d'utiliser la seule section des dettes actives et passives, qui n'est qu'un bilan au décès du livre de comptes du marchand, ni celles des immeubles et des titres et papiers. Les dettes, ne l'oublions pas, tout comme les immeubles sont des signes d'une condition économique à un moment précis du temps. Cependant, il faut souligner que ces informations conviennent parfaitement à une étude partielle, car elles peuvent servir d'indicateurs et parfois illustrer la situation économique du marchand. Ces sections sont assez étoffées, il faut l'admettre, et elles fournissent des statistiques intéressantes. C'est là où s'arrête leur utilisation et où débute aussi la recherche globale

11 Voir à ce sujet les articles de George Bervin, «Espace physique et culture matérielle du marchand-négociant à Québec (1820-1830), op . cit. et «Environnement matériel et activités économiques des conseillers exécutifs et législatifs à Québec, 1810-1830», op. cit. De plus, les professeurs Paquet et Wallot l'ont aussi clairement démontré dans: «Les inventaires après décès à Montréal au tournant du XIX ${ }^{\mathrm{e}}$ siècle: préliminaires à une analyse», op. cit., et dans «Structures sociales et niveaux de richesse dans les campagnes du Québec, 1792-1812», Bulletin d'histoire de la culture matérielle, 17 (Musée national de l'Homme, Ottawa): printemps 1983: 25-44. 
visant à cerner le potentiel économique de l'individu et, logiquement, sa place dans la hiérarchie sociale ${ }^{12}$.

Cette recherche globale nécessite l'utilisation de renseignements provenant de sources variées: inventaires après décès, contrats de mariage, donations, actes de vente de toutes sortes, registres de vente de terres, contrats d'engagement, rentes constituées, obligations, quittances, actes de transport, marchés, procurations, actes de société et affrètements de navires. De plus, l'historien pourra consulter, s'ils sont disponibles, les livres de comptes de l'individu et des correspondances privées. Toutes ces consultations n'ont qu'un but - que l'inventaire après décès seul ne permet pas d'atteindre —, à savoir, de suivre à la trace l'origine, la répartition et la mobilité de la fortune de l'individu visé.

Avant d'évaluer de quelle façon ces différentes sources archivistiques peuvent apporter une contribution déterminante à la recherche historique, nous allons montrer par un exemple qu'il est utile, voire indispensable, d'exploiter une grande variété de documents.

La section des dettes de l'inventaire après décès du marchandnégociant John Goudie révèle une dette active de $4900 £$ et une dette passive de $24340 £$. Ce solde négatif de $19440 £$ laisse croire techniquement que le défunt lègue à ses héritiers un patrimoine en mauvaise posture. Or, en poussant plus loin l'enquête et en utilisant d'autres sources, nous pouvons constater que la situation financière de Goudie est loin d'être alarmante. Par exemple, le registre de la Compagnie d'assurance de Québec contre les accidents du feu ${ }^{13}$ dévoile que Goudie détient plusieurs actions de cette compagnie, au coût de $100 £$ chacune. De plus, les Statuts provinciaux du Bas-Canada montrent qu'il est un des souscripteurs-fondateurs de la Banque de Québec créée en $1818^{14}$, ce qui veut dire qu'il possède de nombreuses actions, à $25 £$ l'unité, de cet établissement financier. Nous n'avons pas trouvé jusqu'à maintenant de documents nous permettant de croire qu'il s'en est départi, et cette constatation vaut aussi pour ses actions dans la Compagnie d'assurance de Québec.

Comme la plupart des grands marchands et des gens de l'élite de la première moitié du XIX ${ }^{\mathrm{e}}$ siècle, Goudie est aussi un grand propriétaire foncier et immobilier. La section des immeubles de son inventaire

\footnotetext{
12 Lorsqu'il est impossible de retracer l'inventaire après décès de certains marchands importants, devons-nous conclure que nous sommes incapables de tracer leur profil économique et social? Nullement, car si nous avons affaire à un personnage clé de l'économie, nous pourrons pallier au manque d'inventaire après décès par l'utilisation de nombreux autres actes notariés le concernant. De plus, ce marchand figurera en bonne place dans la section des dettes actives et passives d'autres commerçants tout aussi importants.

13 ANQ-Q, AP-P-1738 (Fonds privés).

14 Les Statuts provinciaux du Bas-Canada, 11 (1821): c. 26.
} 
après décès nous fait voir clairement cet aspect de ses activités, mais elle n'indique pas la valeur en argent de ses propriétés. Pour en connaître l'étendue, nous avons analysé des actes de vente de terres présentant les mêmes caractéristiques que les siennes, ainsi que les registres de vente de terres par le shérif, que nous étudierons plus loin. Plusieurs des immeubles et des terres de Goudie ont été vendus par le shérif afin de payer de multiples créanciers. En comptabilisant ces ventes, nous constatons que leur total est supérieur au solde négatif inscrit dans la section des dettes.

Et enfin, l'inventaire de Goudie ne met pas en évidence la manière dont il a amassé sa fortune au cours de sa vie active, ni ne tient compte de la variété de ses activités économiques, de même que de son réseau de relations. En regroupant diverses sources, nous sommes donc en mesure de mieux apprécier l'importance du personnage ${ }^{15}$. L'analyse de sources autres que l'inventaire après décès nous permet donc de modifier notre interprétation sur la situation financière réelle de John Goudie et, par le fait même, nous amène à conclure que le défunt n'était pas en faillite au moment de son décès.

L'exemple de Goudie montre d'une certaine façon les difficultés auxquelles est confronté le chercheur traçant une étude socioéconomique des divers groupes présents dans le Bas-Canada. Aucune analyse ou interprétation ne peut être définitive, car l'introduction de nouvelles données peut continuellement modifier notre connaissance du sujet.

Quelles sont donc ces sources accessibles à l'historien de la bourgeoisie marchande de la première moitié du $\mathrm{XIX}^{\mathrm{e}}$ siècle dans le BasCanada? Retenons tout d'abord:

\section{a) L'acte de procuration}

La qualité des informations que renferme l'acte de procuration ${ }^{16}$ (Power of Attorney) en fait un document d'une très grande valeur. L'utilisation d'un bon nombre d'actes de ce type peut susciter des études d'histoire économique et sociale d'un genre nouveau. L'acte de procuration peut être déterminant, par exemple, pour l'identification des réseaux de relations d'un marchand important ou, de façon générale, des couches supérieures. En effet, il permet d'identifier les différentes villes où le mandant détient des intérêts économiques, en plus de cerner

\footnotetext{
15 Actuellement, nous sommes en train de compiler le plus d'actes notariés possible concernant John Goudie, ainsi que d'autres marchands-négociants de Québec (tels les Pozer et William Burns), pour la période allant de 1800 à 1830 . Les résultats nous serviront dans une étude socioéconomique de leurs activités commerciales.

${ }_{16}$ Comme complément d'information sur l'acte de procuration, voir Jean-Paul Poisson, «De quelques nouvelles utilisations des sources notariales en histoire économique $\left(\mathrm{XVII}^{\mathrm{e}}-\mathrm{XX}{ }^{\mathrm{e}}\right.$ siècle)», Revue historique, no 505 (janv.-mars 1973): 5-22, plus particulièrement pages 14-15.
} 
clairement le genre d'activité qu'il y pratique. Les chercheurs pourront alors reconstituer le profil socio-professionnel des commettants et des mandataires.

Dans les minutiers des notaires de la première moitié du XIX ${ }^{\mathrm{e}}$ siècle nous avons repéré deux types principaux d'actes de procuration. Le premier, d'ordre général, se rapporte bien souvent à un individu qui, devant s'absenter du pays pour une assez longue période, «nomme comme procureur spécial» ou «donne pouvoir» à une personne de gérer «ses biens et ses affaires». Cette procuration, quoique générale, est totale en ce sens qu'elle permet à celui qui la reçoit de «recouvrer de l'argent», de «négocier des transactions», d' «intenter des poursuites», en un mot de faire tout ce qu'il jugera utile dans l'intérêt de son commettant. D'autre part, même s'il ne doit pas s'absenter, un individu peut donner une procuration générale à un mandataire. Cela se fait, entre autres, lorsque surgissent des problèmes successoraux. Dans l'acte de procuration passé en pareil cas, quelqu'un (assez souvent un marchand) est désigné comme procureur spécial dans le but de «recevoir toutes les sommes dues à la communauté», de «louer des maisons», de «régler avec les héritiers légataires ou autres prétendants», etc. Ici, le procureur est l'administrateur d'une succession et, à ce titre, il gérera les biens jusqu'à son règlement final.

La deuxième sorte d'acte de procuration s'avère une source plus restreinte, car elle a trait à un motif précis. Dans un tel cas, la procuration peut être de «vendre des intérêts dans le vaisseau Helen» qui est à Glasgow ${ }^{17}$ ou encore de «récupérer une certaine somme d'argent». Elle concerne une opération ponctuelle et est donc plus limitée dans le temps.

Après la compilation de plusieurs actes de procuration nous avons pu dégager quelques constatations. Tout d'abord, ces actes sont généralement d'ordre économique, surtout dans le milieu de la bourgeoisie marchande et des autres groupes aisés. Nous n'avons pas rencontré jusqu'à maintenant de procurations données par un artisan, un journalier, un cultivateur ou un charpentier. La totalité des procurations sont signées par un marchand, un membre des professions libérales ou un haut fonctionnaire de l'État. Il s'agit donc d'un document se rattachant à des groupes sociaux précis en milieu urbain.

La compilation d'actes de procuration permet d'étudier les relations commerciales variées d'un marchand-négociant ou d'une catégorie de marchands. L'acte de procuration passé en vue d'une opération à l'étranger nous semble le plus utile, car il permet de voir l'importance économique du marchand dans le grand commerce et de suivre l'évolution de ses affaires. Ainsi, nous savions que le marchand-négociant

17 Procuration donnée par John Goudie. ANQ-Q, not. Jean Bélanger, 1811. 
et constructeur de navires de Québec John Goudie faisait affaire dans les deux Canadas et en Angleterre. Grâce à un acte de procuration dans lequel Goudie donne pouvoir à Robert Tucker de gérer toutes ses affaires aux «Isles des Bermudes», nous avons réalisé l'ampleur de ses ramifications commerciales à l'étranger.

Les membres de la bourgeoisie marchande, en particulier ceux qui occupent le sommet de la pyramide au cours de la première moitié du $\mathrm{XIX}^{\mathrm{e}}$ siècle, cherchent presque tous à diversifier leurs sphères d'activités économiques en transigeant souvent à l'extérieur du pays. Généralement, lorsque l'acte de procuration concerne une opération effectuée en dehors de la ville où habite le commettant, il est donné à un associé, à un agent commercial ou à un parent. Lorsqu'il s'agit d'une transaction faite à l'étranger, les principaux intéressés sont presque exclusivement des marchands-négociants.

Ajoutons enfin que l'acte de procuration présente malgré tout quelques lacunes pour l'historien. Les chiffres qu'il fournit ne sont pas toujours précis et l'acte conserve dans bien des cas un caractère général. Ainsi, lorsqu'on donne procuration de "gérer tous les biens», nous pouvons nous demander quelle est leur nature et leur importance, et quel pourcentage touche celui qui reçoit la procuration.

\section{b) Le livre de comptes}

Pour l'étude du milieu des affaires, le livre de comptes s'avère un instrument d'analyse efficace. Y apparaissent les multiples transactions commerciales effectuées par un marchand, quelquefois aussi les commandes et les livraisons de marchandises, les ventes de gros et de détail, les noms des créanciers et des débiteurs, de même que les sommes d'argent en jeu ${ }^{18}$. Il existe également des livres de comptes de compagnies qui contiennent des informations d'ordre économique de première qualité. Tel est le cas, par exemple, du registre de la Compagnie d'assurance de Québec contre les accidents du feu ${ }^{19}$, qui renferme la liste des souscripteurs, le nombre d'actions en leur possession ainsi que les dividendes qui leur ont été versés au fil des années. Le livre de comptes du Pont Dorchester, par exemple, où sont consignées toutes les entrées de devises au jour le jour, fournit le nom des individus qui ont acquitté le droit de passage et les sommes d'argent perçues. Les livres comptables de la Banque de Québec ${ }^{20}$ (1818-1831) renferment eux aussi de nombreuses données qui font voir les dépôts et les retraits d'argent faits périodiquement par l'élite économique.

18 L'emploi du livre de compte comme l'une des sources principales d'une étude à caractère historique et économique est assez répandu. Voir en particulier l'article de Louis Michel, «Le livre de compte (1784-1792) de Gaspard Massue, marchand à Varennes», Histoire sociale, 13, 26 (nov. 1980): 369-398.

19 ANQ-Q, AP-P-1738, Quebec Fire Assurance Company.

20 ANQ-Q, Banque de Québec, Ledgers. 
Les dépôts d'archives renferment toute une variété de livres de comptes: livres tenus par un individu ${ }^{21}$ et comptant une dizaine de pages, ceux par exemple plus volumineux de la Banque de Québec qui comprennent en moyenne 500 pages, ou encore les livres des communautés religieuses. Ces derniers renferment de nombreuses informations de toutes sortes, en particulier sur des citoyens importants de la ville qui ont des relations avec ces communautés.

Que doit-on attendre des livres ou des registres de comptes? Il faut d'abord noter que le livre de comptes est un document aride et bref, car on y trouve que des colonnes de noms, de dates, de rentrées et de sorties d'argent. Ces informations sont importantes, mais le chercheur doit posséder déjà d'autres sources d'informations et les comparer avec les données du livre. Malgré tout, le livre ou le registre constitue un instrument de travail essentiel pour reconstituer même en partie le volume des activités commerciales d'un marchand, son stock de marchandises, le nombre de personnes composant sa clientèle et leur répartition géographique, et aussi la période de l'année la plus fructueuse au plan de son chiffre d'affaires. Il permet également de connaître le nombre de ses employés et leur salaire, de savoir si le marchand fait affaire sur le plan régional, national et international. Ce document nous livre également certaines informations à caractère social. À partir des noms qui y figurent il est possible de reconstituer partiellement le réseau d affaires du marchand et de se faire une opinion sur l'importance de ce dernier par rapport au groupe dont il est issu.

\section{c) L'acte de vente de terres et d'immeubles et le registre de vente de terres par le shérif}

Les actes de vente de terres et d'immeubles, utilisés en série et étalés sur plusieurs périodes, permettent de suivre la progression et la répartition géographique de la richesse foncière d'un marchand par exemple, tout en nous renseignant sur la valeur vénale de ses propriétés. Ils aident aussi à établir un indice du taux d'inflation sur les propriétés foncières concernées.

Ces actes nous amènent à constater le phénomène de la spéculation foncière, qui constitue l'un des traits dominants de l'économie de la première moitié du $\mathrm{XIX}^{\mathrm{e}}$ siècle bas-canadien. Certaines propriétés changent de mains très rapidement à l'époque, surtout en milieu urbain. La façon courante de procéder du spéculateur consiste à acheter une vaste étendue de terre en ville ou à la périphérie et à la revendre par la suite sous forme de lots. Cette pratique (achat, fractionnement de la terre et vente simple ou à constitution de rente) entraîne un profit sub-

\footnotetext{
21 Il n'est pas toujours facile de trouver dans les archives le livre de comptes d'un marchand, car il se trouve souvent parmi d'autres documents.
} 
stantiel pour le grand spéculateur foncier. Il est donc utile de dépouiller le plus grand nombre d'actes de vente possible et de ne pas se fier à la seule section des immeubles de l'inventaire après décès, qui indique seulement les biens fonciers possédés par l'individu à sa mort.

À partir d'une compilation massive d'actes de vente, il devient possible de dresser le prix d'achat moyen des maisons et des lots dans une région donnée ${ }^{22}$. Cette compilation permet de classer les acheteurs en fonction de catégories socio-professionnelles et de l'origine ethnique $^{23}$. La préparation d'une échelle des prix pour des édifices localisés dans un même secteur nécessite beaucoup de temps et de ressources matérielles. On ne retrace pas rapidement des actes de vente se rattachant à des maisons présentant des caractéristiques semblables, quant aux dimensions du terrain, à la localisation, au matériau et à l'architecture. On ne construit pas une moyenne à partir d'une dizaine d'actes de vente, mais après en avoir dépouillé une grande quantité, et lorsque cette moyenne est établie, on peut déterminer la valeur marchande relative des biens fonciers et immobiliers ou, à tout le moins, obtenir un ordre de grandeur valable. De même, l'analyse d'un échantillon assez considérable de ces actes permet d'identifier les groupes sociaux ${ }^{24}$ qui détiennent le monopole de la propriété foncière en milieu urbain et rural ${ }^{25}$ dans la première moitié du XIX ${ }^{\mathrm{e}}$ siècle. Les actes de vente permettent de mettre en relief une certaine concentration de la propriété foncière, particulièrement en milieu urbain ${ }^{26}$. Une observation rapide nous montre que dans la haute ville de Québec, entre 1800 et 1830, la plupart des grandes propriétés sont acquises soit par les marchands-négociants, soit par les hauts fonctionnaires de l'État, ou encore, par certains membres des professions libérales.

Les registres de vente de terres par le shérif ${ }^{27}$ constituent une source complémentaire essentielle à l'acte de vente, en vue de l'établissement

22 On peut aussi à partir des marchés de construction avoir de nombreuses informations sur le coût de construction des résidences. À ce sujet, voir les marchés de construction passés chez les notaires de la région de Québec entre 1800 et 1870 , tels que répertoriés par G. Bastien et al., dans Inventaires des marchés de construction des archives civiles de Québec, 1800-1870 (3 vols., Parcs Canada, Ottawa, 1975). Voir aussi l'article de Jacques Bernier, «La construction domiciliaire à Québec, 1810-1820», RHAF, 31, 4 (mars 1978): 547-561 .

23 Le rôle d'évaluation de la ville fournit presque le même type d'informations pour ces deux derniers points. Il donne le nom du propriétaire, les noms des locataires ainsi que leur profession (Archives de la ville de Québec), Série CC (Finances), Rôles d'évaluation et d'imposition, 1821-1829 (microfilms).

24 Nous excluons de ces groupes sociaux les communautés religieuses qui sont de grands propriétaires fonciers, tant à la ville qu'à la campagne, de même que les autorités militaires. À ce dernier sujet, consulter l'article de Claudette Lacelle, «La propriété militaire à Québec de 1760 à 1871», Collection Histoire et archéologie, 57 (Parcs Canada, Ottawa, 1982): 152-249.

${ }_{25}$ Concernant la question de la propriété foncière dans certaines zones rurales du BasCanada, se rapporter à l'article de Fernand Ouellet, «La sauvegarde des patrimoines dans le district de Québec durant la première moitié du XIX ${ }^{\mathrm{e}}$ siècle», RHAF, 26, 3 (déc. 1972): 319-374.

26 Dans ce cas-ci, il s'agit surtout de la ville de Québec et de sa périphérie, pour laquelle nous avons effectué le dépouillement.

27 ANQ-Q, Registres des ventes par le shérif, 1824-1927. 
d'une moyenne des coûts approximatifs des biens fonciers et immobiliers à l'achat. Après l'annonce faite à l'avance dans les journaux, ces terrains ou ces bâtisses sont vendus à l'encan par le shérif sur ordre de la Cour du Banc du roi. Dans les registres, on trouve le numéro de la cause inscrite devant la Cour, les noms du plaignant et du défendeur, la date d'inscription de la cause et finalement la date d'adjudication et le montant de la vente avec le nom de l'acheteur. Sur le plan économique, une observation attentive des registres nous révèle que les lots, les édifices et les terrains les meilleurs sont acquis lors de l'adjudication par des gens aisés. Autrement dit, les propriétés les plus rentables et les mieux capitalisables à long terme sont achetées par des membres de la bourgeoisie. Lorsque le prix demandé par le shérif au moment de la vente d'un lot ou d'une maison est inférieur au prix réel du marché, on perçoit rapidement le profit réalisé par l'acheteur. Du point de vue social, les registres nous fournissent le nom de l'acheteur, le lieu de sa résidence et parfois sa profession. Toutes ces données judicieusement compilées permettront à l'historien de dresser un tableau précis du profil social de l'acheteur.

À notre avis, toute nouvelle recherche historique portant sur la propriété foncière et immobilière dans l'une ou l'autre des deux grandes villes du Bas-Canada, dans la première moitié du XIX ${ }^{\mathrm{e}}$ siècle, doit tenir compte de ces sources.

\section{d) L'acte d'affrètement de navire}

L'acte appelé affrètement de navire (Charter party) est indispensable à celui qui s'intéresse au commerce international dans la première moitié du XIX ${ }^{\mathrm{e}}$ siècle. (Voir l'annexe I). Il met en lumière toute la structure du déroulement des opérations dans le circuit du commerce national et international. Ainsi, il nous permet de savoir qui loue le navire, le salaire que cette personne paye aux membres d'équipage, de même que les différentes conditions et obligations qui régissent le transport maritime à l'époque. Selon nous, les informations les plus importantes ont trait à la (ou aux) destination(s) du navire affrété ainsi qu'aux marchandises transportées.

L'acte d'affrètement de navire est un document essentiel pour qui veut connaître les activités d'un marchand dans «un ensemble assez complexe d'échanges internationaux ${ }^{28}$ et les différents secteurs économiques à l'intérieur desquels il mène ses affaires. Grands importateurs et exportateurs, les marchands-négociants ne sont pas tous de simples intermédiaires entre les commerçants locaux et ceux des grandes villes du Royaume-Uni, même si à l'occasion ils peuvent être les

\footnotetext{
28 Gilles Paquet et Jean-Pierre Wallot, «Aperçu sur le commerce international et les prix domestiques dans le Bas-Canada (1793-1812)», RHAF, 21, 3 (déc. 1967): 450.
} 
deux à la fois. Cet acte peut nous apprendre, par exemple, qu'un grand marchand de Québec a loué un navire pour faire transporter et aussi pour prendre livraison de marchandises dans des endroits aussi éloignés que Gibraltar, Cadiz, Lisbonne et Liverpool; qu'un autre a expédié une cargaison de bois à Saint-Jean, Terre-Neuve.

Par ailleurs, les actes d'affrètement de navire ${ }^{29}$ montrent que lorsqu'il s'agit de transactions à caractère international, les individus concernés se font souvent payer ou payent eux-mêmes en lettres de change négociables à Londres, à Liverpool ou dans d'autres grandes cités commerciales ${ }^{30}$. Les marchands-négociants bas-canadiens connaissent donc les rouages des relations financières de leur époque, en plus d'être devenus un maillon important du réseau d'affaires OutreAtlantique.

L'acte d'affrètement de navire, qui concerne presque uniquement les grands marchands, permet d'analyser les multiples transactions effectuées par ces derniers au cours de la première moitié du XIX siècle. La quantité des données qu'il nous livre permet d'ajouter des détails supplémentaires à l'étude des moyens d'enrichissement de la grande bourgeoisie marchande bas-canadienne.

\section{e) Les sources reliées au crédit}

De nombreux autres documents notariés méritent également une exploitation approfondie. Certains peuvent fournir des éléments essentiels à une histoire des relations économiques et sociales en milieu urbain. Ainsi, l'acte d'obligation et l'acte de quittance (voir les annexes II et III) peuvent être déterminants dans les recherches portant sur les activités commerciales et le crédit à l'intérieur d'une ville. Avec les données qu'ils contiennent, ils peuvent mener par exemple à l'identification des grands créanciers actifs dans cette ville et conséquemment de ceux qui ont la mainmise sur les différentes activités commerciales ${ }^{31}$. Leur utilisation met en relief de larges pans de la structure et des méca-

29 Les marchands-négociants sont aussi bien des locataires que des locateurs de navires. Parfois même ceux qui possèdent des navires en louent d'autres propriétaires afin d'expédier des marchandises. Par exemple, nous avons remarqué que le marchand-négociant et constructeur de navires John Goudie a quelquefois affrété un navire pour une opération économique précise. Il a aussi loué un navire au gouvernement afin que celui-ci puisse rapatrier ses troupes dans la métropole.

30 Les lettres de change sont aussi des documents sérieux pour connaitre et évaluer les activités d'un marchand ou d'une société commerciale sur le plan du commerce international. À cet égard, Gilles Paquet et Jean-Pierre Wallot ont mis en évidence l'importance des lettres de change dans les transactions entre les grands marchands, actifs à l'intérieur et à l'extérieur du BasCanada. Les auteurs mentionnent des lettres de change tirées par des marchands bas-canadiens et négociables à New York ou en Angleterre, pour des sommes aussi élevées que $20000 £$, (Gilles Paquet et Jean-Pierre Wallot, «Le système financier bas-canadien», L'Actualité économique revue d'analyse économique, 59, 3 (sept. 1983): 456-513).

31 À ce sujet, voir la deuxième partie, qui porte sur le crédit et son fonctionnement dans la ville de Québec, de l'étude de George Bervin, «Aperçu sur le commerce et le crédit à Québec, 1820-1830», op. cit.: 540-551. 
nismes du crédit dans la première moitié du XIX $\mathrm{X}^{\mathrm{e}}$ siècle dans le BasCanada. Ils sont d'autant plus importants que la plupart des prêts d'argent ou autres formes d'endettement se font en présence du notaire. Ces actes nous font voir que malgré l'apparition des banques en 1817 et 1818 , le crédit demeure une affaire personnalisée, c'est-à-dire que des individus s'endettent envers d'autres individus.

Une analyse de ces deux actes peut permettre également de repérer les membres des couches urbaines les plus aisées. Ainsi, nous avons constaté que les débiteurs de sommes d'argent élevées (plus de $1000 £$ ) sont presque tous issus du milieu des grands commercants, des professions libérales ou encore du groupe des hauts fonctionnaires de l'État. De plus, les deux contrats mentionnés renferment de multiples détails qui indiquent assez souvent les raisons de l'endettement (achat de marchandises, acquisition d'un bien immobilier, versement direct d'argent, achat ou construction d'un navire), qui sont généralement d'ordre commercial, surtout lorsque la somme est élevée. Sur le plan social, ils permettent de mesurer l'importance du créancier, autrement dit sa notabilité, puisqu'on est à même d'identifier l'éventail de sa clientèle. Il peut être possible aussi de connaître ses activités sur le plan extérieur, car il est courant de rencontrer des obligations dont le remboursement sera effectué, par exemple, en Angleterre ou dans le Haut-Canada. Mais malgré leur richesse, ces documents ont aussi leurs limites, dans la mesure où certains d'entre eux peuvent ne pas répondre à notre attente, en mentionnant seulement que le prêt a été accordé «dès avant la présente» signature de l'acte notarié.

D'autres sources peuvent aussi convenir à une étude du système du crédit et des réseaux d'affaires dans le Bas-Canada. Parmi elles, retenons les billets de reconnaissance de dettes qui ne nécessitent pas la présence du notaire. Dans ces billets, le débiteur reconnaît devoir un montant $\mathrm{x}$ à son créancier, sans généralement apporter d'autres précisions. Nous avons constaté que les deux parties concernées passent par la suite chez le notaire pour transformer le billet en obligation. Cette rencontre chez le notaire peut se faire après un délai assez long.

Nous pouvons compter aussi sur l'acte de transport, dont le résumé qui suit nous fait voir les multiples facettes du prêt d'argent à cette époque.

Fut présent Monsieur Jacob Pozer, marchand, demeurant à Québec, lequel a cédé et transporté... à Monsieur Charles Jourdain Mte Maçon, à Québec aussi y demeurant à ce présent et acceptant la somme de Six mille livres du cours de Québec à lui dû par le sieur Frederick Pomerman marchand demeurant à Montréal, suivant l'obligation que ce dernier en a passée au dit Jacob Pozer par devant notaires à Montréal le deuxième jour de septembre [1820]... aussi transporté les intérêts échus et à échoir sur la dite somme, à 
l'effet duquel le dit Jacob Pozer a présentement délivré au dit Charles Jourdain la première expédition de la dite obligation... Ce transport fait moyennant et pour demeurer quitte par le dit Jacob Pozer envers le dit Charles Jourdain de pareille somme de Six mille livres courant qu'il lui doit... Fait à Québec le neuvième jour de septembre mil huit cent vingt. ${ }^{32}$

L'acte de transport s'apparente donc à l'acte d'obligation, l'unique différence entre les deux étant que le débiteur change de créancier. Dans ce document, que l'on rencontre fréquemment dans les archives notariales, toutes les clauses de l'ancienne obligation demeurent inchangées entre le nouveau créancier et le débiteur. Quant à savoir quels motifs poussent le prêteur à transférer la dette en faveur d'un nouveau créancier, aucune indication n'apparaît dans l'acte.

\section{Conclusion}

L'objectif de cette étude était de jeter un éclairage nouveau sur la valeur et les limites de certaines sources utilisées pour faire l'histoire économique de l'élite marchande bas-canadienne. Vu l'ampleur de la documentation disponible dans les dépôts d'archives, on comprendra qu'il aurait été pour le moment difficile d'en faire un tour d'horizon complet.

Ainsi donc, il reste un travail énorme à effectuer afin de faire connaître le contenu et l'utilité pour l'historien des nombreux autres documents qui n'ont pas été analysés à l'intérieur de ce texte. À cet égard, cette étude doit être vue comme une première phase dans notre tentative qui vise à long terme à identifier la quasi-totalité des sources archivistiques pouvant servir à explorer les secteurs économiques des marchands-négociants bas-canadiens. Malgré tout, l'historien intéressé par des questions socio-matérielles et économiques touchant la bourgeoisie marchande trouvera des informations pertinentes en consultant les documents d'archives mentionnés dans ce texte et, à partir de cellesci, il sera en mesure d'ajouter des éléments neufs à l'interprétation historique.

Nous croyons avoir fait ressortir le fait que, s'ils sont utilisés séparément, la plupart de ces actes notariés ne peuvent permettre des interprétations définitives sur un aspect donné, car ils ne réunissent pas en eux-mêmes toute l'information souhaitée. D'où l'importance de la jonction de plusieurs actes notariés avec d'autres sources (tels les imprimés de l'époque et les sources iconographiques), sans laquelle il peut être difficile de se lancer dans une étude axée par exemple sur le groupe des marchands-négociants.

32 ANQ-Q, not. Thomas Lee, 1820. Le Charles Jourdain dont il est question dans le présent acte n'est pas un artisan. En réalité, c'est un gros entrepreneur qui fait des affaires avec les membres de la haute bourgeoisie, dont quelques-uns lui doivent de très fortes sommes. 
En effet, il faut comprendre que cette catégorie sociale, dont la caractéristique première réside dans la diversification de ses activités économiques, utilise toute une série de moyens, pas toujours facilement repérables, pour maximiser son capital et s'enrichir. C'est la raison pour laquelle il est important de multiplier les investigations en vue de mieux cerner le comportement de cette élite sociale et économique du Bas-Canada.

La connaissance du contenu des documents archivistiques nous apparaît donc indispensable à l'élaboration de monographies historiques se rattachant à une catégorie sociale qui en son temps et à sa façon a influencé le cours de l'histoire.

\section{ANNEXE I \\ Exemple d'acte d'affrètement de navire ${ }^{33}$}

By this Charter party or affreightment between Jacob Poser of Quebec in the province of Lower Canada, merchant, freighter of the brig or vessel called the Little Belt of Quebec, whereof James Wilson is now master of the burthen of 173 tons or thereabouts, and now laying in the port of Quebec on the one part and Messrs Jones, White \& Melvin also of Quebec aforesaid merchants, acting for and on the part \& behalf of Henry Harbeck, now of Montreal, merchant, charterer of the said brig or vessel called the Little Belt, by this present Charter party, on the other part. It is mutually agreed that the said vessel, being tight, stounch, manned, victualled and every way fitted for the voyage, shall receive on board from the said Jones, White \& Melvin acting as aforesaid a full and compleat cargo of wheat, or any other lawful merchandise or goods as the said vessel can conveniently stow and carry her cabin and sufficient room for the crew; water, tackle and provisions excepted and the said vessel when soladen, shall proceed with the first opportunity of wind weather to Gibraltar, and on her way call at Cadiz for orders if required so to do by the charterer or his agent; and when so safely arrived at Gibraltar, shall remain there five days, which are not to be counted as part of the lay days; after which shall proceed with all possible speed to Cadiz, Lisbon, Liverpool, or any other admissible port in St George's Channel, or either of said ports, if required, where a full true delivery of said cargo shall be made in good order, agreeable to Charter party and Bills of lading; and so end the said intended voyage, restraint of princes and rulers of the seas and navigation, fire, pirates, and enemies excepted during said voyage and that the said vessel shall have for the purpose of discharging at the port of delivery, fifteen working days, to reckon from her arrival and being placed in a suitable birth and the master of said ship having given due notice to the charterer's agent or consignee of the said ship being ready to discharge the said cargo agreeably to Bills of lading,

\footnotetext{
33 ANQ-Q, not. Edward Glackmeyer, 13 juin 1812.
} 
the said fifteen days not to be commence untill after the five days allowed to the consignee to determine whether the vessel is to unload there or proceed to Lisbon, Cadiz or any other port as before mentioned.

In consideration whereof the said Jones, White \& Melvin acting as aforesaid promise, engage, and agree to load the said vessel with a full \& compleat cargo of wheat or other lawful merchandise or good, as aforesaid to be brought along side within the reach of the vessel's tackels, and also to receive or cause to be received the same at the port of delivery. And also shall \& will pay or cause to be paid the said Jacob Poser, the sum of two shillings and nine pence sterling for each and every Winchester bushel, delivered in good order at Gibraltar provided the charterer or his agent requests that the cargo should be there discharged and which the owner and master agrees to, and an addition of four pence sterling shall be made, if the vessel returns from Gibraltar to unload at Lisbon or Cadiz, and a further addition of ten pence sterling shall be made for each and every Winchester bushel delivered at Liverpool or any other port in St George's Channel, but should the vessel proceed from Gibraltar direct to Liverpool or any admissible port in St George's Channel then and in that case the freight is to be paid at the rate of three shillings and seven pence sterling for each and every Winchester bushel, together with the sum of five pounds sterling thereon per centum to stand in lieu of all pilotage and port charges, that shall or may occur during the said intended voyage. The payement of all which said sums shall be made in good bills of exchange at par, payable in London at thirty days sight or in spanish dollars at the rate of four shillings and six pence sterling each to remain at the option of the said charterer or his assigns, and also shall and will pay or cause to be paid the demurrage if any at the rate of six guineas per day for each and every day of said vessels detention, over $\&$ above the days before mentioned allowed to discharge said cargo. It is also agreed between the parties that the said charterer or consignee shall pay to the owner, agent or master of said ship the sum of two hundred pounds sterling in specie or cash at the port of delivery, to be paid at the same rate of premium or discount as good bills of exchange at thirty days sight on London are curently selling there at the time; which said sum of two hundred pounds sterling is to be accounted as part of the freight to become due to the owner of said vessel and further that all dunnage, mats, shifting and lining boards and all \& every other materials necessary to line the said vessel shall be provided for, and made by the said charterer and at their own costs and expences, and shall by them be taken out of the said vessel at the port of delivery. It is further mutually agreed that a suitable and sufficient room shall be allowed in the said vessel's cabin for the charterer's agent or supercargo and passage allowed him, from hence to the port of delivery free of any charge he furnishing himself with provisions \& bedding. And for the true execution of the present Charter party the said parties bind themselves respectively each unto the other in the penal sum of one thousand pounds current money of Quebec firmly by these presents.

In witness whereof the said parties have to these presents set and subscribed their names this thirteenth day of June one thousand eight hundred and twelve.

(Suivent les signatures) 


\section{ANNEXE II \\ Exemple d'acte d'obligation ${ }^{34}$}

Pardevant les notaires publics pour la province du Bas-Canada résidens à Québec soussignés. Fut présent Mr John Reinhart tabaconiste de la ville de Québec lequel a reconnu \& confessé devoir bien légitimement à Mr François Lehoullier, marchand résident en la dite ville de Québec, à ce présent \& acceptant créancier, \& retenant pour lui ses hoirs \& ayans cause à l'avenir, c'est à savoir, la somme de quatre mille livres du cours actuel de cette province, pour prêt de pareille somme que le dit François Lehoullier lui a réellement comptée, nombrée $\&$ délivrée en espèces ayant cours en cette dite province, dès avant l'exécution des présentes - dont quittance.

Laquelle dite somme de quatre mille livres susdit cours le dit débiteur, promet \& s'oblige, pour lui ses hoirs \& ayans cause, payer, compter \& délivrer au dit créancier ses dits hoirs $\&$ ayans cause, comme suit savoir la somme de deux mille livres susdit cours en trois années de cette date et pareille somme de deux mille livres résidu de la dite somme principale en cinq années de cette date pour tout délais, \& avec l'intérêt légal sur la dite somme à compter de la date des présentes jusqu'au parfait payement, le dit intérêt payable \& acquittable au dit créancier ses hoirs \& ayans cause de six mois en six mois, \& dont le premier payement écherra $\&$ se fera le quatrième jour de décembre prochain $\&$ ainsi continuer de six mois en six mois jusqu'au remboursement de la dite somme capitale, mais lequel intérêt diminuera au prorata de chaque payement fait comme susdit.

Et pour sûreté de laquelle dite somme capitale de quatre mille livres susdit cours \& des intérêts qui en proviendront le dit débiteur a affecté, obligé \& hypothéqué tous ses biens généralement quelconques présens \& futurs.

Et pour l'exécution des présentes le dit débiteur fait élection de domicile irrévocable en sa demeure actuelle. Par ainsi promettant, obligeant \& renonçant.

Fait \& passé à Québec en l'étude de L. T. Macpherson l'un des notaires soussignés. L'an mil huit cent dix neuf le quatrième jour de juin après midi. Et ont les dites parties signé, de ce requises, avec nous dits notaires. Lecture faite, vingt mots rayés sont nuls et deux renvois en marge bons.

(Suivent les signatures)

\section{ANNEXE III Exemple d'acte de quittance ${ }^{35}$}

Pardevant les notaires publics pour la province du Bas-Canada soussignés fut présent l'honorable John Caldwell écuier membre des Conseils Exécutif \& Législatif \& receveur général de Sa Majesté en cette province. Lequel sur

${ }_{34}$ ANQ-Q, not. L. T. Macpherson, 4 juin 1819.

35 ANQ-Q, not. Louis Panet, 13 oct. 1823. 
réquisition de John Goudie écuier de la cité de Québec, à ce présent et acceptant, a volontairement fait la déclaration suivante, savoir. Que sur à compte et en déduction de la somme de deux mille livres courant à lui dus par le dit John Goudie pour balance du prix de l'Hôtel de Lauzon circonstances \& dépendances, situés en la paroisse St. Joseph de la Pointe Lévy, suivant acte de vente passé à Québec devant les Notaires dont Mtre [...] l'un d'eux a gardé minute en date du [...] mil huit cent [...] lui le dit John Caldwell a eu et reçu du dit John Goudie divers effets, fournitures et marchandises au montant de la somme de quatre cent cinquante sept livres neuf shillings un denier et demi courant suivant compte réglé entre eux \& imputé sur la dite balance d'après leur conventions expresses et de plus une somme de six cent quarante trois livres douze chelins courant pour autant payé par le dit John Goudie pour et à l'acquit de Messrs Caldwell et Davidson, à la requisition du dit John Caldwell, suivant et au désir d'un acte passé entre John White et Francis Languedoc d'une part, et les dits Caldwell et Davidson et le dit John Goudie d'autre part, devant les Notaires à Québec et dont Mtre Têtu l'un deux a gardé minute en date du quatre de février mil huit cent vingt. Au moyen desquels payemens et imputations la balance due au dit John Caldwell par le dit John Goudie sur le prix de vente du dit Hôtel de Lauzon \& ses dépendances se trouve réduite à la somme de huit cent quatre vingt seize livres dix huit chelins et dix deniers et demi courant, exclusivement des intérêts. Desquelles déclaration \& reconnaissance, les dits comparans nous ayant requis acte, nous leur avons octroyé le présent pour leur servir et valoir, ce que droit. À Québec en l'étude de Mtre Louis Panet l'un de nous dits Notaires, l'an mil huit cent vingt trois le treize octobre avant midi, les dits comparans ayant signé après lecture faite trois mots rayés sont nuls.

(Suivent les signatures) 